\title{
Occurrence of lysogenic bacteria in marine microbial communities as determined by prophage induction
}

\author{
Sunny C. Jiang, John H. Paul* \\ Marine Science Department, University of South Florida, 1407 th Ave. South, St. Petersburg, Florida 33705, USA
}

\begin{abstract}
Viruses are abundant and dynamic members of the marine microbial community, and it is important to understand their role in the ecology of natural microbial populations. We have previously found lysogenic bacteria to be a significant proportion $(43 \%)$ of the cultivable heterotrophic microbial population. As the majority of marine bacteria are not cultivable using standard plating methods, we measured the proportion of marine lysogenic bacteria in natural communities by prophage induction. Mitomycin C, UV radiation, sunlight, temperature and pressure were used to induce prophage in lysogenic bacteria from estuarine, coastal and oligotrophic offshore environments. To determine if hydrocarbon pollutants may cause the induction of marine lysogens, aromatic and aliphatic hydrocarbons (including Bunker $\mathrm{C} \# 6$ fuel oil, phenanthrene, naphthalene, pyrene, and trichloroethylene) were also used as inducing agents. Induction was most often found in estuarine environments, where viral direct counts increased from 128.8 to $345 \%$ of the uninduced control, resulting in mortality of 10.5 to $67.3 \%$ (average $34 \%$ ) of the bacterial population. Up to $38 \%$ of the bacterial population was lysogenized in estuarine environments, as calculated from an average burst size. Microbial populations from oligotrophic offshore environments were inducible at 3 of 11 stations sampled. Eight of the 11 samples $(73 \%)$ treated with polyaromatic hydrocarbons resulted in prophage induction in natural populations. Time series analysis was also conducted in 2 samples induced by mitomycin $C$ from the Atlantic Ocean near the coast of North Carolina, USA. For both samples, significant decreases in bacterial numbers were detected in treated samples after 8 h of incubation. A significant increase of viruses was detected at $8 \mathrm{~h}$ at one station and at $24 \mathrm{~h}$ at the other station after induction. This study indicates that natural lysogenic populations are sensitive to a variety of inducing agents, and induction occurs more frequently in coastal and estuarine environments than offshore environments.
\end{abstract}

KEY WORDS: Virus B Bacteria Lysogen - Marine microbial community - Induction

\section{INTRODUCTION}

Lysogens are bacteria that contain a silent viral genome. The viral DNA is termed a prophage, and replicates during host cell division. The prophage can become active spontaneously to vegetative replication and produce viral particles or can be induced to lytic viral production by chemical, physical and other agents (Ackermann \& DuBow 1987). In the marine environment, lysogeny may be one of the strategies for viruses to survive periods of low host density and/or nutrient depletion (Freifelder 1987). On the other

- Addressee for correspondence.

E-mail: jpaul@seas.marine.usf.edu hand, lysogeny has been shown to benefit the host for a variety of reasons, including homoimmunity, conversion by phage genomes and increased general fitness (Levin \& Lenski 1983).

Viruses are the most abundant microorganisms in the oceans. The density of viruses ranges from $\sim 10^{4}$ particles $\mathrm{ml}^{-1}$ in oligotrophic and deep sea environments to over $10^{8}$ particles $\mathrm{ml}^{-1}$ in estuarine and coastal environments (Børsheim 1993, Fuhrman \& Suttle 1993, Weinbauer et al. 1995). Recent studies suggest that viruses are dynamic members of the microbial community and that they play an important role in controlling bacteria and phytoplankton mortality in the ocean (Fuhrman \& Suttle 1993, Cottrell \& Suttle 1995, Fuhrman \& Noble 1995, Weinbauer \& Peduzzi 1995). 
However, many of these studies also indicated some degree of uncertainty on the viral contribution to carbon flow in the microbial loop ecosystem, in some cases overestimating the virus-induced mortality of microbial populations (Bratbak et al. 1992). Many scientists suggest that in the marine environment, the final fate of viral infection may be lysogenization rather than lysis (Bratbak et al. 1990, 1992, Heldal \& Bratbak 1991, Thingstad et al. 1993). A study of viral production and bacterial mortality in microcosms suggested that lytic viral production was the major means of viral regeneration and bacterial mortality rather than prophage induction in lysogens (Wilcox \& Fuhrman 1994). However, these results do not conflict with the hypothesis that many bacteria in the marine environment may be lysogenic and therefore homoimmune (i.e. resistant to lytic viral infection).

In a previous study we demonstrated that $43 \%$ of the marine bacterial isolates examined were inducible by mitomycin C (Jiang \& Paul 1994). Since less than $1 \%$ of marine bacteria are cultivable on conventional media, these results may not be representative of the lysogenic population in natural communities of marine bacteria. Moreover, previous studies on marine (Jiang \& Paul 1994) and freshwater (Tapper \& Hicks 1994) lysogens only used artificial inducing agents, such as mitomycin $\mathrm{C}$ and $\mathrm{UV}$ radiation $(<300 \mathrm{~nm})$, neither of which are found in the marine environment. The environmental factors which cause prophage induction in the marine environment are unknown. Here we report the induction of indigenous lysogenic bacteria from a variety of marine environments using mitomycin $\mathrm{C}_{1}$ UV radiation, sunlight, temperature, pressure and aromatic and aliphatic hydrocarbons as inducing agents

\section{MATERIAL AND METHODS}

Sampling sites. Water samples were collected during 2 research cruises: in the Gulf of Mexico during June 1994, on the RV 'Pelican', and in the Atlantic Ocean during July 1995, on the RV 'Cape Hatteras'. Station locations for the Pelican and Cape Hatteras cruises are indicated in Fig. $1 \mathrm{~A} \& 1 \mathrm{~B}$, respectively. Subsurface and deep water samples were taken with 201 Niskin bottles, and surface waters were collected by pumping directly into acid-washed $20 \mathrm{l}$ carboys. All samples were processed immediately. Water samples from Mamala Bay, Oahu, Hawaii, USA, were also used for this study. Samples in Hawaii were taken from sites in Pearl Harbor, autside the mouth of Pearl Harbor, off shore of Ala Wai Canal and offshore of Diamond Head. Samples were processed at the University of Hawaii within $3 \mathrm{~h}$ of sampling.

Induction of lysogenic bacteria by chemical and physical inducing agents. Of each water sample, 10 to 100 I was concentrated by a Membrex Vortex Flow filtration system to a final volume of 40 to $60 \mathrm{ml}$ as previously described (Paul et al. 1991, Jiang et al. 1992). The concentrated samples were subdivided and treated either by adding mitomycin $\mathrm{C}\left(1 \mu \mathrm{g} \mathrm{m} \mathrm{m}^{-1}\right)$, exposing to $254 \mathrm{~nm}$ wavelength UV radiation (NIS G15T8 $15 \mathrm{~W}$ germicidal light) for $30 \mathrm{~s}\left(14.76 \mathrm{~mJ} \mathrm{~cm}{ }^{-2}\right)$ in a sterile petri-dish $\left(5 \mathrm{ml}\right.$ dish $\left.^{-1}\right)$, exposing to sunlight for $15 \mathrm{~min}$. in a petri-dish on the deck with or without UV blocking (Commercial Plastic Co., Clearwater, FL, USA), subjecting to increased temperature or pressure, or left untreated (control). Except for the Gulf of Mexico Stn 4' and Stn 9' samples, temperature experiments were conducted by exposing $5 \mathrm{ml}$ Membrex-concen-
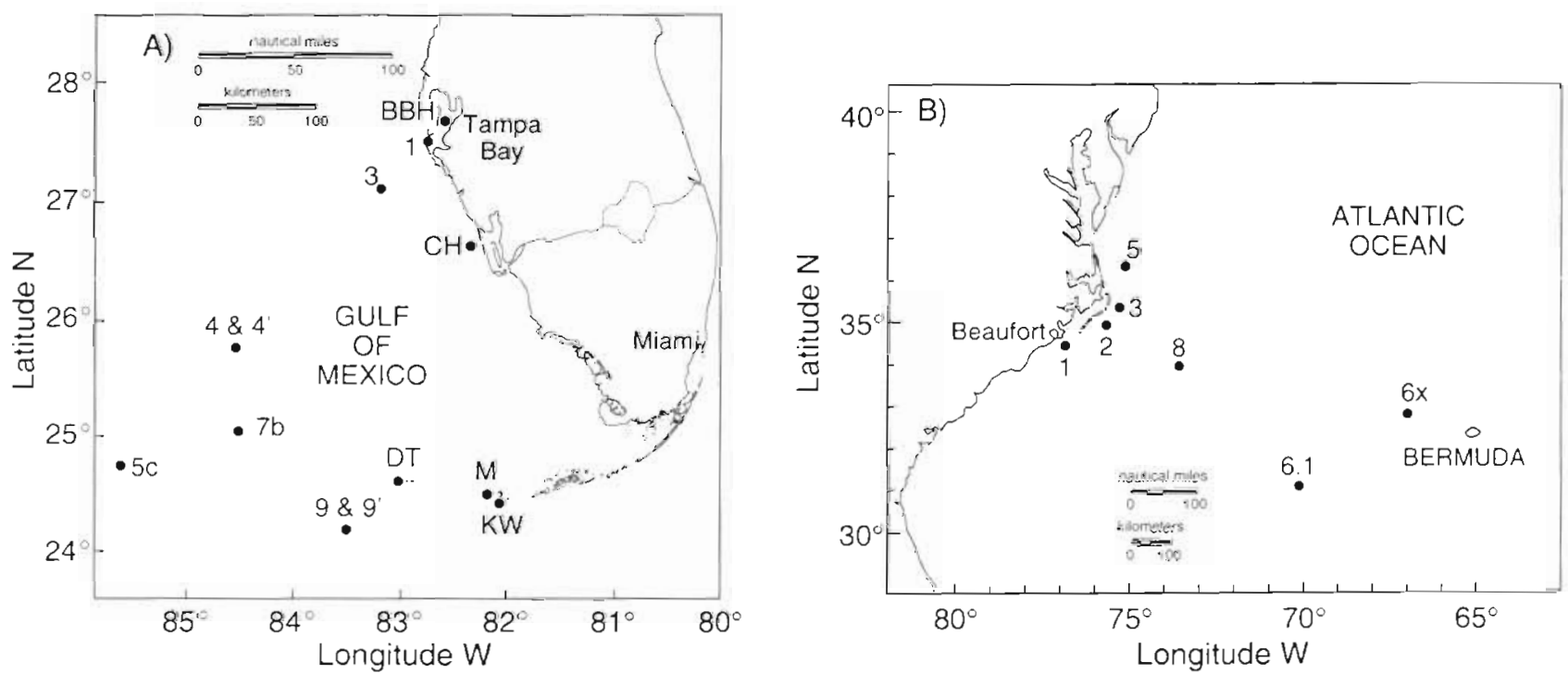

Fig. 1. Sampling locations. (A) Gulf of Mexico during June 1994. BBH: Bayboro Harbor; CH: Charlotte Harbor; DT: Fort Jefferson Mote, Dry Tortugas; M: Marquesas; KW: Key West Harbor; (B) Atlantic Ocean during July 1995 
trated samples to 30,37 or $42^{\circ} \mathrm{C}$ for $30 \mathrm{~min}$, then incubating for $16 \mathrm{~h}$ at $24^{\circ} \mathrm{C}$. The control was kept at $24^{\circ} \mathrm{C}$. For the Gulf of Mexico Stn 4' and Stn 9' samples, freshly collected waters from $250 \mathrm{~m}$ subsurface $\left(14^{\circ} \mathrm{C}\right)$ were left in a shady area on the deck overnight. The final temperature of the water sample was $30^{\circ} \mathrm{C}$ before concentration by Membrex the next morning The control samples were kept at $10^{\circ} \mathrm{C}$ in a cold room. Pressure experiments were performed by lowering the Membrex-concentrated sample in sealed, screw-cap microfuge tubes to between 827 and $2000 \mathrm{~m}$ depth and maintaining in that environment for at least $30 \mathrm{~min}$. All samples were fixed with $2 \%$ glutaraldehyde immediately after 16 to $24 \mathrm{~h}$ incubation and stored at $4^{\circ} \mathrm{C}$ for bacterial direct counts (BDC) and viral direct counts (VDC). Samples which had significantly increased viral direct counts and significantly decreased bacteria direct counts were considered lysogenic inductions.

Induction of lysogenic bacteria by aromatic and aliphatic hydrocarbons. Membrex-concentrated oligotrophic offshore samples from Atlantic Ocean and unconcentrated nearshore station water samples from the coast of North Carolina, USA, were used for this study. Bunker $C$ \#6 fuel oil (Texaco Inc.), phenanthrene, naphthalene and pyrene (Chem Service, West Chester, PA, USA) were each dissolved in hexane (Fisher Chemical, Pittsburg, PA) to make a stock solution. The organic solvent was then evaporated in the fume hood after the desired amount of chemical was added to clean amber glass bottles, leaving only chemicals in the bottle. Water samples collected from different stations were added to the chemical-containing amber bottles on the cruise. Each sample contained $50 \mu \mathrm{g} \mathrm{ml} \mathrm{m}^{-1}$ naphthalene, phenanthrene or pyrene, or

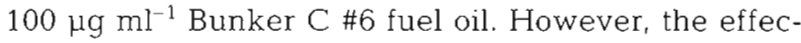
tive concentration based on solubility at $25^{\circ} \mathrm{C}$ and $35 \%$ salinity was 22.5 and $0.72 \mu \mathrm{g} \mathrm{ml}^{-1}$ for naphthalene and phenanthrene, respectively (Eganhouse \& Calder 1976). The solubility of pyrene at $25^{\circ} \mathrm{C}$ and $35 \%$ salinity was calculated to be $0.08 \mu \mathrm{g} \mathrm{ml}^{-1}$, based on its solubility at 0\% salinity (May \& Wasik 1978). Solubility for Bunker $C$ \#6 fuel oil in seawater was unknown. Trichloroethylene (a liquid solution) was added to the water sample for a final concentration of $4 \% \mathrm{v} / \mathrm{v}$. All samples were well mixed and incubated in the dark for $24 \mathrm{~h}$ before fixing with glutaraldehyde.

Induction time series. A final concentration of $1 \mu \mathrm{g}$ $\mathrm{ml}^{-1}$ mitomycin $\mathrm{C}$ was added to a $500 \mathrm{ml}$ water sample in a plastic $1 \mathrm{l}$ flask. A replicate flask without mitomycin $\mathrm{C}$ was used as control. Both flasks were incubated in the dark. Forty $\mathrm{ml}$ of each sample was taken each time from both flasks at $0,4,8$ and $24 \mathrm{~h}$. The samples were fixed for viral direct counts and bacterial counts.

Viral and bacterial direct counts. VDC for Membrex-concentrated water samples were performed as previously described by Paul et al. (1991), except a new Hitachi 7100 transmission electron microscope (TEM) was used. Viral numbers in unconcentrated water samples were enumerated by the ultracentrifugation method of Bergh et al. (1989). Two grids of each sample were prepared. Virus-like particles were counted in 2 windows (opening on the grid) from each grid and 30 randomly selected fields in each window at 50000 times magnification directly from the TEM screen. Pictures of bacteria containing mature phage were taken at magnification of 30000 to 60000 times.

Membrex-concentrated samples were diluted with $0.2 \mu \mathrm{m}$ filtered artificial seawater for BDC by epifluorescent microscopy (Paul 1982). BDC were also performed with a TEM at 10000 times magnification for unconcentrated samples using the same grid for VDC.

Statistical analyses. Multi-sample comparison by analysis of variance and 2-sample comparison by t-test were performed using Statgraphics software (Manugistics Inc., Rockville, MD, USA) and further comparison between control and each treatment was performed using Dunnett's test (Zar 1984). Statistical comparisons for VDC were generated from the average of 4 grid-windows counts of each sample. Statistical comparisons for BDC were generated from 3 replicate slides of each sample.

\section{RESULTS}

\section{Induction of lysogenic bacteria from marine environments}

Fig. 2 and Table 1 show the results of the induction of indigenous lysogenic bacteria from eutrophic estuarine environments. Mitomycin C, UV radiation, elevated temperature and sunlight were tested as inducing agents. Five of 7 environments sampled showed a significant increase in VDC in response to one or more inducing agents. VDC in the induced samples ranged from 128.8 to $345.8 \%$ of the control samples. Most samples that showed a significant increase in VDC also showed significant decreases of BDC (58.4 to $89.5 \%$ of the controls). Our criterion for induction was a significant increase in VDC concomitant with a significant decrease in BDC. The sixth and the seventh samples (Key West Harbor and Pearl Harbor) showed increases in VDC (140 to $170 \%)$ that were not statistically significant owing to variability in $\mathrm{VDC}$, even though decreases in BDC were noted.

The effect of sunlight on the microbial community varied, including no significant effect on the Gulf of Mexico Stn 1 sample, increases in both VDC and BDC in the Marquesas sample, and decreases in both VDC and $\mathrm{BDC}$ in the Fort Jefferson Mote sample. Increasing 


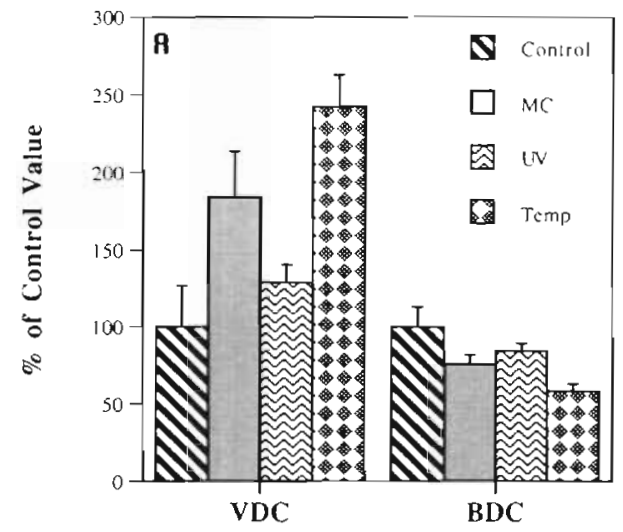

temperature from 24 to $42^{\circ} \mathrm{C}$ for $30 \mathrm{~min}$ also caused the induction of viruses $(242 \%$ of the control) and decreases in BDC (58.4\% of the control) in a Bayboro Harbor sample. Assuming the increased viral numbers and

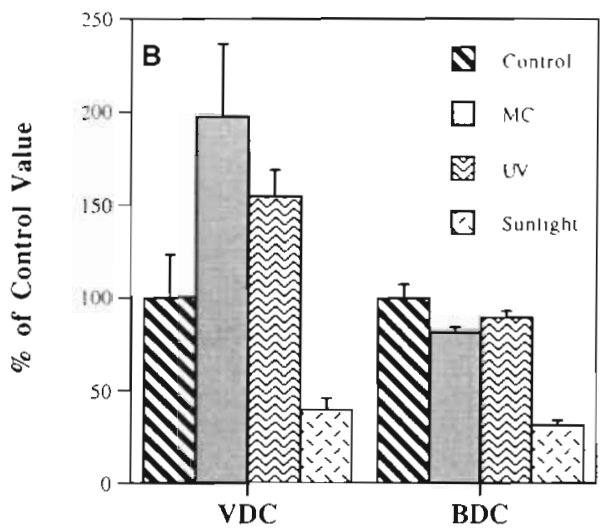

Fig. 2. Induction of natural communities of marine lysogens by mitomycin $C$. UV radiation, temperature and sunlight in samples collected from (A) Bayboro Harbor and (B) Fort Jefferson Mote. Error bars for VDC generated from the average counts of each of 4 windows in 2 replicate grids, and for BDC generated from counts from 3 replicate slides

decreased bacteria in the induced samples were solely caused by lysogenic induction, from 10.5 to $41.6 \%$ of the bacteria in these samples were inducible lysogens. The estimated burst size for lysogenic bacteria in these

Table 1. Induction of indigenous lysogenic bacteria from eutrophic estuarine environments by chemical and physical inducing agents. For viral and bacterial direct counts, values for controls are given as virus or bacteria per ml respectively, values for inducing agents as \% of control. Significance (S) and insignificance (I) at $95 \%$ confidence; na: not applicable

\begin{tabular}{|c|c|c|c|c|}
\hline Sample & $\begin{array}{l}\text { Viral direct count } \\
\text { (control: virus } \mathrm{ml}^{-1} \\
\text { agents: \% of control) }\end{array}$ & $\begin{array}{l}\text { Bacterial direct count } \\
\text { (control: bacteria } \mathrm{ml}^{-1} \text {; } \\
\text { agents: \% of control) }\end{array}$ & $\begin{array}{c}\% \text { of lysogen } \\
\text { Bacterial } \\
\text { mortality }\end{array}$ & $\begin{array}{l}\text { Acteria based on } \\
\text { Average } \\
\text { burst size }(30)\end{array}$ \\
\hline \multicolumn{5}{|l|}{ Bayboro Harbor } \\
\hline Control & $1.45 \pm 0.39 \times 10^{7}$ & $3.90 \pm 0.52 \times 10^{6}$ & & \\
\hline Mitomycin C & $184.2(\mathrm{~S})$ & $75.9(\mathrm{~S})$ & 24.1 & 10.4 \\
\hline UV light & $128.8(\mathrm{~S})$ & $84.6(\mathrm{~S})$ & 15.4 & 3.5 \\
\hline Temperature $\left(42^{\circ} \mathrm{C}, 30 \mathrm{~min}\right)$ & 242.5 (S) & $58.4(\mathrm{~S})$ & 41.6 & 17.7 \\
\hline \multicolumn{5}{|c|}{ Gulf of Mexico Stn $1\left(27^{\circ} 35^{\prime} \mathrm{N}, 82^{\circ} 43^{\prime} \mathrm{W}\right)$} \\
\hline Control & $1.84 \pm 0.24 \times 10^{7}$ & $3.00 \pm 0.05 \times 10^{6}$ & & \\
\hline Mitomycin C & $165(\mathrm{~S})$ & $64 \quad(S)$ & 36 & 13.3 \\
\hline UV light & $286(\mathrm{~S})$ & $61.5(5)$ & 38.5 & 38.0 \\
\hline Sunlight & $132(\mathrm{I})$ & 123 (S) & na $a^{b}$ & na \\
\hline \multicolumn{5}{|l|}{ Marquesas } \\
\hline Control & $2.7 \pm 0.84 \times 10^{\mathrm{h}}$ & $8.11 \pm 0.35 \times 10^{5}$ & & \\
\hline Mitomycin C & $242.8(\mathrm{~S})$ & $66.6(S)$ & 33.4 & 15.8 \\
\hline UV light & $345.8(\mathrm{~S})$ & $72.6(S)$ & 27.4 & 27.3 \\
\hline Sunlight & $597.8(\mathrm{~S})$ & $379.8(S)$ & na & na \\
\hline \multicolumn{5}{|l|}{ Key West Harbor } \\
\hline Control & $4.13 \pm 0.7 \times 10^{6}$ & $7.09 \pm 0.33 \times 10^{5}$ & & \\
\hline Mitomycin C & 154.2 (I) & $84.5(5)$ & na & na \\
\hline UV light & $170.2(\mathrm{I})$ & $72.1(\mathrm{~S})$ & na & na \\
\hline \multicolumn{5}{|l|}{ Fort Jefferson Mote } \\
\hline Control & $1.15 \pm 0.27 \times 10^{7}$ & $2.67 \pm 0.2 \times 10^{\mathrm{b}}$ & & \\
\hline Mitomycin C & $197.4(\mathrm{~S})$ & $81.3(S)$ & 18.7 & 14.0 \\
\hline UV light & $154.8(\mathrm{~S})$ & $89.5(\mathrm{~S})$ & 10.5 & 7.9 \\
\hline Sunlight & 39.7 (S) & $31.4(5)$ & na & na \\
\hline \multicolumn{5}{|l|}{ Pearl Harbor, Hawail } \\
\hline Control & $1.24 \pm 0.24 \times 10^{5}$ & $1.9 \pm 0.2 \times 10^{6}$ & & \\
\hline Mitomycin C & $140.3(\mathrm{I})$ & $62.6(S)$ & na & na \\
\hline \multicolumn{5}{|l|}{ Mouth of Pearl Harbor, Hawaii } \\
\hline Control & $7.36 \pm 2.92 \times 10^{5}$ & $1.47 \pm 0.06 \times 10^{5}$ & & \\
\hline Mitomycin $C$ & $217.7(\mathrm{~S})$ & $65.3(5)$ & 34.7 & 2 \\
\hline
\end{tabular}


Table 2. Induction of indigenous lysogenic bacteria from marine coastal environments by chemical and physical inducing agents. For viral and bacterial direct counts, values for controls are given as virus or bacteria per ml respectively, values for inducing agents as \% of control. Significance (S) and insıgnificance (I) at $95 \%$ confidence; na: not applicable

\begin{tabular}{|c|c|c|c|c|}
\hline Sample & $\begin{array}{l}\text { Viral direct count } \\
\text { (control: virus } \mathrm{ml}^{-1} \\
\text { agents: } \% \text { of control) }\end{array}$ & $\begin{array}{l}\text { Bacterial direct count } \\
\text { (control: bacteria ml }{ }^{1} \\
\text { agents: } \% \text { of control) }\end{array}$ & $\begin{array}{c}\% \text { of lysoger } \\
\text { Bacterial } \\
\text { mortality }\end{array}$ & $\begin{array}{c}\text { acteria based on } \\
\text { Average } \\
\text { burst size (30) }\end{array}$ \\
\hline \multicolumn{5}{|l|}{ Charlotte Harbor } \\
\hline Control & $1.06 \pm 0.31 \times 10^{6}$ & $3.25 \pm 0.33 \times 10^{5}$ & & \\
\hline Mitomycin C & $75.9(1)$ & $77.8(\mathrm{~S})$ & na & na \\
\hline UV light & $117.9(1)$ & 75.9 (S) & na & na \\
\hline \multicolumn{5}{|c|}{ Atlantic Stn $2\left(35^{\circ} 00.25^{\prime}, 75^{\circ} 34.5^{\prime}\right)$} \\
\hline Control & $2.62 \pm 0.54 \times 10^{5}$ & $9.69 \pm 0.60 \times 10^{5}$ & & \\
\hline \multicolumn{5}{|l|}{ Temperature } \\
\hline $30^{\circ} \mathrm{C}, 30 \mathrm{~min}$ & $269.8(S)$ & $73.5(\mathrm{~S})$ & 26.5 & 1.5 \\
\hline $37^{\circ} \mathrm{C}, 30 \mathrm{~min}$ & $178.6(\mathrm{I})$ & $76.4(\mathrm{~S})$ & na & na \\
\hline $42^{\circ} \mathrm{C}, 30 \mathrm{~min}$ & 198.1 (I) & $68.3(\mathrm{~S})$ & na & na \\
\hline \multicolumn{5}{|c|}{ Atlantic Stn $5\left(36^{\circ} 36^{\prime} \mathrm{N}, 75^{\circ} 06^{\prime} \mathrm{N}\right)$} \\
\hline Control & $2.35 \pm 0.47 \times 10^{6}$ & $8.20 \pm 0.50 \times 10^{5}$ & & \\
\hline Pressure (2000 m, $30 \mathrm{~min})$ & $122.6(\mathrm{I})$ & $100(\mathrm{I})$ & na & na \\
\hline \multicolumn{5}{|c|}{ Offshore of Ala Wai Canal, Hawaij } \\
\hline Control & $9.15 \pm 4.66 \times 10^{4}$ & $3.75 \pm 0.3 \times 10^{5}$ & & \\
\hline Mitomycin C & $77.6(1)$ & $54.1(S)$ & na & na \\
\hline
\end{tabular}

samples, calculated by dividing increased VDC by decreased BDC ( $\triangle \mathrm{VDC} / \triangle \mathrm{BDC}$ ), ranged from 1.7 to 30 .

The second approach for estimating the percentage of lysogens in bacterial communities is to assume an average burst size and calculate the number of lysogens by dividing increased viruses with the burst size. From 11 randomly taken pictures of bacteria containing mature phage we have determined that lysogenic induction burst sizes ranged from 6 to 142 , averaging 38.3. Since this data was generated from a relatively small sample size and because we did not conduct a systematic study of burst size by TEM examination, we also took into consideration burst sizes from lysogenic induction of natural populations by other researchers (M. G. Weinbauer \& C. A. Suttle pers. comm.). The average burst size from this communication and our own data was 30 . The percentage of lysogenic bacteria calculated by this average burst size ranged from 2 to $38 \%$ in estuarine environments.

The results of induction of lysogenic bacteria from marine coastal environments are shown in Table 2 . Of the 4 samples tested, only the sample from Atlantic Stn 2 showed induction in VDC (269.8\% of the control) and a significant decrease in BDC $(73.5 \%$ of the control) after an elevated temperature treatment. Slight viral increases were also found in the UV-treated offshore Charlotte Harbor sample and pressure-treated Atlantic Stn 5 sample, yet these were not significant when statistically compared with the control. Statistically significant decreases of BDC were detected in nearly all treated samples, except for the Atlantic sample subjected to the increased pressure.

Compared with the samples from estuarine environments, the number of inducible lysogens in oligotrophic offshore environments was significantly lower (Table 3). Samples collected from 11 stations were tested using mitomycin C, UV radiation, natural sunlight, temperature and pressure as inducing agents. Only 2 samples, from Gulf of Mexico Stn $7 \mathrm{~b}$ and Atlantic Stn 6.1, showed significant increase in VDC and decrease in BDC numbers after induction by UV radiation or mitomycin $C$, respectively. Significant increases in VDC were also found in the pressuretreated Gulf of Mexico Stn 9 sample and the UVtreated Atlantic Stn 6x sample, yet no significant changes in BDC could be detected. Increases in VDC were also found in the mitomycin $\mathrm{C}$-treated Gulf of Mexico Stn 4 sample, and the UV-treated and temperature-treated Gulf of Mexico Stn 9 ' samples. However, these increases were not statistically significant. Using a UV-blocking plastic cover to filter out UV radiation from the natural sunlight did not make a difference in BDC or VDC compared to the sample with a UV-transparent cover. Pressure experiments were performed twice, at Gulf of Mexico Stn 4 and Gulf of Mexico Stn 9. In both experiments, there were increases in VDC, but no significant changes in BDC.

Aliphatic and aromatic hydrocarbons, including Bunker $\mathrm{C} \# 6$ fuel oil, phenanthrene, naphthalene, pyrene and trichloroethylene, were used to induce 
lysogenic bacteria in samples from the Atlantic Ocean in both Membrex concentrated and unconcentrated samples (Fig. 3, Table 4). No prophage induction could be detected in any of the samples treated by Bunker C \#6 fuel oil. However, phenanthrene, naphthalene and pyrene showed significant induction (i.e. increases in

Table 3. Induction of indigenous lysogenic bacteria from oligotrophic offshore environments by chemical and physical inducing agents. For viral and bacterial direct counts, values for controls are given as virus or bacteria per mil respectively, values for inducing agents as \% of control. Signuficance (S) and insignificance (I) at $95 \%$ confidence; na: not applicable

\begin{tabular}{|c|c|c|c|c|}
\hline \multirow[t]{2}{*}{ Sàmple } & \multirow{2}{*}{$\begin{array}{l}\text { Viral direct count } \\
\text { (control: virus } \mathrm{ml}^{-1} \\
\text { agents: \% of control) }\end{array}$} & \multirow{2}{*}{$\begin{array}{l}\text { Bacterial direct count } \\
\text { (control: bacteria } \mathrm{ml}^{-1} \\
\text { agents: } \% \text { of control) }\end{array}$} & \multicolumn{2}{|c|}{$\%$ of lysogenic bacteria based on } \\
\hline & & & $\begin{array}{l}\text { Bacterial } \\
\text { mortality }\end{array}$ & $\begin{array}{c}\text { Average } \\
\text { burst size }(30)\end{array}$ \\
\hline \multicolumn{5}{|c|}{ Gulf of Mexico Stn $3\left(27^{\circ} 04^{\prime} \mathrm{N}, 83^{\circ} 18^{\prime} \mathrm{W}\right)$} \\
\hline Control & $1.78 \pm 0.66 \times 10^{5}$ & $3.69 \pm 0.2 \times 10^{5}$ & & \\
\hline Mitomycin C & $139(I)^{\mathrm{d}}$ & $78.3(\mathrm{~S})$ & na & na \\
\hline \multicolumn{5}{|c|}{ Gulf of Mexico Stn $4\left(25^{\circ} 38.63^{\prime} N, 84^{\circ} 35.63^{\prime} \mathrm{W}\right)$} \\
\hline Control & $1.86 \pm 1.24 \times 10^{5}$ & $3.53 \pm 0.13 \times 10^{5}$ & & \\
\hline Mitomycin C & 59.1. (I) & $28.6(S)$ & na & na \\
\hline UV light & 73.7 (I) & 38.5 (S) & na & na \\
\hline Sunlight & $34.9(\mathrm{~S})$ & 39.7 (S) & na & na \\
\hline Pressure $(827 \mathrm{~m}, 30 \mathrm{~min})$ & $142.5(\mathrm{I})$ & $103.1(1)$ & na & na \\
\hline \multicolumn{5}{|c|}{ Gulf of Mexico Stn $4^{\prime}$, subsurface $250 \mathrm{~m}\left(25^{\circ} 38.63^{\prime} \mathrm{N}, 84^{\circ} 35.63^{\prime} \mathrm{W}\right)$} \\
\hline Control & $2.06 \pm 1.17 \times 10^{4}$ & $2.2 \pm 0.04 \times 10^{4}$ & & \\
\hline Temperature $\left(30^{\circ} \mathrm{C}\right.$, overnight $)$ & $71.3(\mathrm{I})$ & $91.8(1)$ & na & na \\
\hline \multicolumn{5}{|c|}{ Gulf of Mexico Stn $5 \mathrm{c}$, deep sea, $1500 \mathrm{~m},\left(24^{\circ} 55^{\prime} \mathrm{N}, 85^{\circ} 32^{\prime} \mathrm{W}\right)$} \\
\hline Control & $8.29 \pm 7.18 \times 10^{3}$ & $1.21 \pm 0.15 \times 10^{4}$ & & \\
\hline Mitomycin C & $87.5(\mathrm{I})$ & 25 (S) & na & na \\
\hline UV light & $52.4(\mathrm{I})$ & $36.4(\mathrm{~S})$ & na & na \\
\hline Sunlight & 102 (I) & $56.6(\$)$ & na & na \\
\hline \multicolumn{5}{|c|}{ Gulf of Mexico Stn $7 \mathrm{~b}$, chlorophyll a max. $\left(25^{\circ} 04^{\prime} \mathrm{N}, 84^{\circ} 27^{\prime} \mathrm{W}\right)$} \\
\hline Control & $7.3 \pm 1.96 \times 10^{4}$ & $1.04 \pm 0.02 \times 10^{5}$ & & \\
\hline Mitomycin C & $194.5(\mathrm{~S})$ & 101 & na & na \\
\hline UV light & 259 (S) & $81.0(\mathrm{~S})$ & 19 & 3.7 \\
\hline Sunlight & $320 \quad(\mathrm{~S})$ & $93.3(\mathrm{I})$ & na & na \\
\hline \multicolumn{5}{|c|}{ Gulf of Mexico Stn 9, chlorophyll a max. $\left(24^{\circ} 14^{\prime} \mathrm{N}, 83^{\circ} 38.8^{\prime} \mathrm{W}\right)$} \\
\hline Control & $1.01 \pm 0.57 \times 10^{5}$ & $1.43 \pm 0.05 \times 10^{5}$ & & \\
\hline Mitomycin C & $98.0(1)$ & $11.9(S)$ & na & na \\
\hline Sunlight & $8.4(\mathrm{~S})$ & $10.1(\mathrm{~S}\}$ & na & na \\
\hline Pressure $(1014 \mathrm{~m}, 30 \mathrm{mmn})$ & 206.9 (S) & $92.3(1)$ & na & na \\
\hline \multicolumn{5}{|c|}{ Gulf of Mexico Stn $9^{\prime}$, subsurface $250 \mathrm{~m}\left(24^{\circ} 14^{\prime} \mathrm{N}, 83^{\circ} 38.8^{\prime} \mathrm{W}\right)$} \\
\hline Control & $6.22 \pm 4.36 \times 10^{4}$ & $9.62 \pm 0.22 \times 10^{3}$ & & \\
\hline UV light & 153 (1) & $74.9(S)$ & na & na \\
\hline Temperature $\left(30^{\circ} \mathrm{C}\right.$, overnight $)$ & $150.3($ (h) & $278.6(\$)$ & na & na \\
\hline \multicolumn{5}{|c|}{ Atlantic Stn $6 \mathrm{x}$, chlorophyll a $\max .\left(33^{\circ} 00^{\prime} \mathrm{N}, 68^{\circ} 00^{\prime} \mathrm{W}\right)$} \\
\hline Control & $4.98 \pm 1.08 \times 10^{5}$ & $1.95 \pm 0.08 \times 10^{5}$ & & \\
\hline IV light & $1357(S)$ & $1010(\mathrm{~T})$ & na & na \\
\hline Sunlight (UV block) & $53.2(S)$ & $91.8(1)$ & na & na \\
\hline (UV trans.) & $62.2(S)$ & $93.8(\mathrm{~d})$ & na & na \\
\hline \multicolumn{5}{|c|}{ Atlantic Stn 6.1 , chlorophyll a max. $\left(31^{\circ} 11^{\prime} \mathrm{N}, 70^{\circ} 18^{\prime} \mathrm{W}\right)$} \\
\hline Control & $4.17 \pm 1.20 \times 10^{5}$ & $2.20 \pm 0.10 \times 10^{5}$ & & \\
\hline Mitomycın C & $166.7(\mathrm{~S})$ & $82.7\{\mathrm{~S} \mid$ & 17.3 & 4.2 \\
\hline \multicolumn{5}{|c|}{ Atlantic $\operatorname{Stn} 8$, chlorophyll a max. $\left(33^{\circ} 59^{\prime} \mathrm{N}, 73^{\circ} 33^{\prime} \mathrm{W}\right)$} \\
\hline Control & $9.06 \pm 2.43 \times 10^{5}$ & $2.84 \pm 0.23 \times 10^{5}$ & & \\
\hline UV light & $97($ l) & $78.2(\mathrm{~S})$ & na & na \\
\hline Sunlight (UV block) & $55.2(\mathrm{I})$ & $76.8(\mathrm{~S})$ & na & na \\
\hline (UV trans.) & $69.4(\mathrm{I})$ & 71.1 (S) & na & na \\
\hline \multicolumn{5}{|c|}{ Offshore of Diamond Head, Oahu, Hawail } \\
\hline Control & $6.51 \pm 2.39 \times 10^{4}$ & $3.01 \pm 0.3 \times 10^{b}$ & & \\
\hline Mitomycin C & $753(\mathrm{I})$ & $73.3(\mathrm{I})$ & na & na \\
\hline
\end{tabular}


Fig. 3. Induction of natural communities of marine lysogens by aliphatic and aromatic hydrocarbons: (A) Sample from Atlantic Stn 1, (B) Sample collected from Atlantic Stn 3. Error bars were generated as in Fig. 2
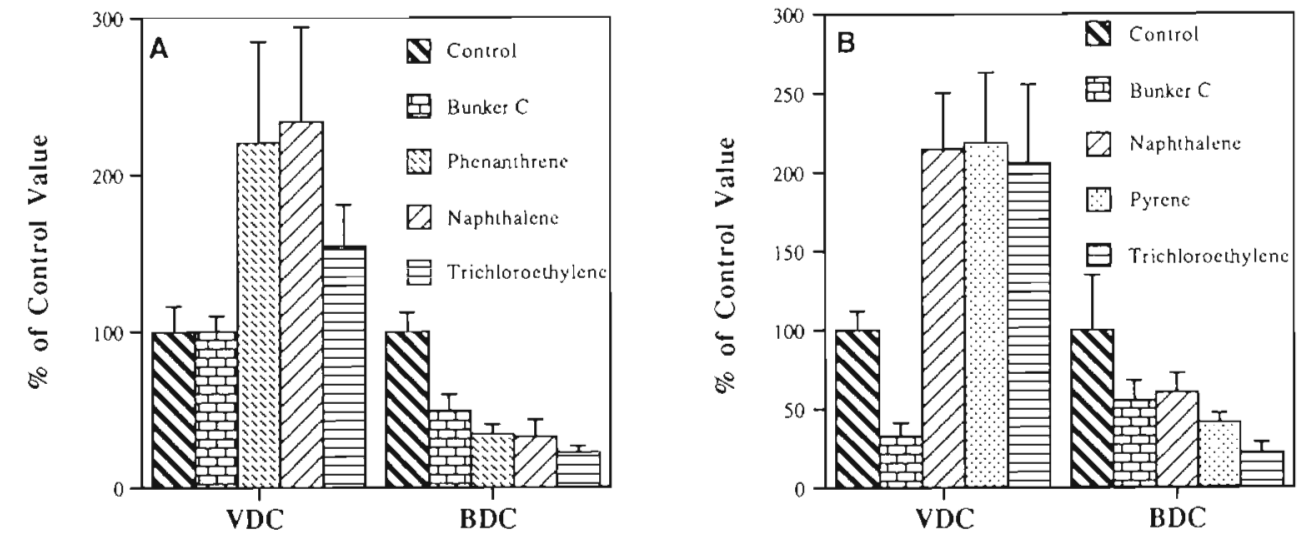

Table 4. Induction of indigenous lysogenic bacteria from a variety of marine environments by aromatic and aliphatic hydrocarbons. For viral and bacterial direct counts, values for controls are given as virus or bacteria per ml respectively, values for inducing agents as \% of control. Significance (S) and insignificance (1) at 95\% confidence; na: not applicable

\begin{tabular}{|c|c|c|c|c|}
\hline Sample & $\begin{array}{l}\text { Viral direct count } \\
\text { (control: } 10^{6} \mathrm{ml}^{-1} \\
\text { agents: \% of control) }\end{array}$ & $\begin{array}{l}\text { Bacterial direct count } \\
\text { (control: } 10^{6} \mathrm{ml}^{-1} \\
\text { agents: } \% \text { of control) }\end{array}$ & $\begin{array}{c}\% \text { of lysogen } \\
\text { Bacterial } \\
\text { mortality }\end{array}$ & $\begin{array}{c}\text { acteria based on } \\
\text { Average } \\
\text { burst size (30) }\end{array}$ \\
\hline \multicolumn{5}{|l|}{ Estuarine environment } \\
\hline \multicolumn{5}{|c|}{ Atlantic Stn $1\left(34^{\circ} 35^{\prime} \mathrm{N}, 76^{\circ} 37^{\prime} \mathrm{W}\right)$} \\
\hline Control & $6.12 \pm 0.97$ & $6.94 \pm 0.83$ & & \\
\hline Bunker C & $100.3(\mathrm{I})$ & $49.4(\mathrm{~S})$ & na & na \\
\hline Phenanthrene & $220.6(\mathrm{~S})$ & $34.4(\mathrm{~S})$ & 65.6 & 3.5 \\
\hline Naphthalene & $233.7(\mathrm{~S})$ & $32.7(\mathrm{~S})$ & 67.3 & 3.9 \\
\hline Trichloroethylene & $154.6(1)$ & $22.5(S)$ & nà & na \\
\hline \multicolumn{5}{|l|}{ Coastal environment } \\
\hline \multicolumn{5}{|c|}{ Atlantic $\operatorname{Stn} 3\left(35^{\circ} 24.5^{\prime} \mathrm{N}, 75^{\circ} 18^{\circ} \mathrm{W}\right)$} \\
\hline Control & $5.26 \pm 0.64$ & $5.99 \pm 2.07$ & & \\
\hline Bunker C & $32.3(\mathrm{~S})$ & $54.8(\mathrm{~S})$ & na & na \\
\hline Naphthalene & $214.4(\mathrm{~S})$ & $60.4(S)$ & 39.6 & 3.3 \\
\hline Pyrene & $218.4(\mathrm{~S})$ & $40.9(\mathrm{~S})$ & 59.1 & 3.5 \\
\hline Trichloroethylene & 205.9 (S) & 21.7 (S) & 78.3 & 3.1 \\
\hline \multicolumn{5}{|c|}{ Atlantic $\operatorname{Stn} 5\left(36^{\circ} 36^{\prime} \mathrm{N}, 75^{\circ} 06^{\prime} \mathrm{W}\right)$} \\
\hline Control & $3.07 \pm 0.48$ & $6.04 \pm 1.19$ & & \\
\hline Bunker C & 65.0 (I) & $36.8(\mathrm{~S})$ & na & na \\
\hline Phenanthrene & 132.9 (I) & $15.6(S)$ & na & na \\
\hline Pyrene & $254.1(\mathrm{~S})$ & 34.7 (S) & 65.3 & 2.6 \\
\hline Trichloroethylene & $65.0(1)$ & $55.6(\mathrm{~S})$ & na & na \\
\hline \multicolumn{5}{|c|}{ Ofíshore environment (induction in Membrex-concentrated water sample) } \\
\hline \multicolumn{5}{|c|}{ Atlantic Stn $6 x$, chlorophyll a max. $\left(33^{\circ} 00^{\prime} N, 68^{\circ} 00^{\prime} W\right)$} \\
\hline Control & $0.5 \pm 0.1$ & $0.2 \pm 0.01$ & & \\
\hline Bunker C & $53.0(\mathrm{~S})$ & $103.6(1)$ & na & na \\
\hline Trichloroethylene & $41.2(\mathrm{~S})$ & $81.5(\mathrm{~S})$ & na & na \\
\hline \multicolumn{5}{|c|}{ Atlantic Stn 6.1, chlorophyll a max. $\left(31^{\circ} 11^{\prime} \mathrm{N}, 70^{\circ} 18^{\prime} \mathrm{W}\right)$} \\
\hline Control & $0.42 \pm 0.12$ & $0.22 \pm 0.01$ & & \\
\hline Bunker C & $126.1(\mathrm{l})$ & $77.3(\mathrm{~S})$ & na & na \\
\hline Phenanthrene & $160.7(S)$ & $86.8(5)$ & 1.3 .2 & 3.9 \\
\hline Naphthalene & $158.8(\mathrm{~S})$ & $86.8(S)$ & 13.2 & 3.7 \\
\hline Pyrene & $229.0(\mathrm{~S})$ & $104.5(\mathrm{I})$ & na & na \\
\hline Trichloroethylene & $51.6(1)$ & $45.0(S)$ & n.a & na \\
\hline \multicolumn{5}{|c|}{ Atlantic Stn 8 , chlorophyll a max. $\left(33^{\circ} 59^{\prime} \mathrm{N}, 73^{\circ} 33^{\prime} \mathrm{W}\right)$} \\
\hline Control & $0.91 \pm 0.24$ & $0.28 \pm 0.02$ & & \\
\hline Bunker C & 124.7 (I) & $79.9(\mathrm{~S})$ & na & na \\
\hline Phenanthrene & $166.7(\mathrm{~S})$ & $75.0(\mathrm{~S})$ & 25 & 7.0 \\
\hline Pyrene & $140.2(1)$ & $88.0(\mathrm{~S})$ & na & na \\
\hline Trichloroethylene & $57.2(\mathrm{I})$ & $51.1(\mathrm{~S})$ & na & na \\
\hline
\end{tabular}


VDC and decreases in BDC) in 8 of the 11 samples treated with these chemicals. VDC in the induced samples ranged from 160.7 to $254 \%$ of the controls. Trichloroethylene was found to induce lysogenic viral production in 1 of the 6 experiments performed. Fig 3 shows representative results for induced samples from Atlantic Stn 1 and Stn 3. At both stations, increases in VDC were found in phenanthrene, naphthalene, pyrene and trichloroethylene treated samples. Decreases in BDC were found in all treated samples. Among all induced samples from a variety of marine environments, from 13.2 to $78.3 \%$ of the bacterial population was killed by these xenobiotic pollutants. However, we cannot separate viral lysis caused by prophage induction from mortality caused by toxicity (see 'Discussion'). The calculation of percentage of lysogens based on the average burst size suggests that only 2.6 to $7 \%$ of the total bacteria population in these environments are lysogens. Subtracting these numbers from the total mortality caused by inducing agent, 9.5 to $75.2 \%$ of the bacteria mortality might be caused by the toxic effect of these chemicals.

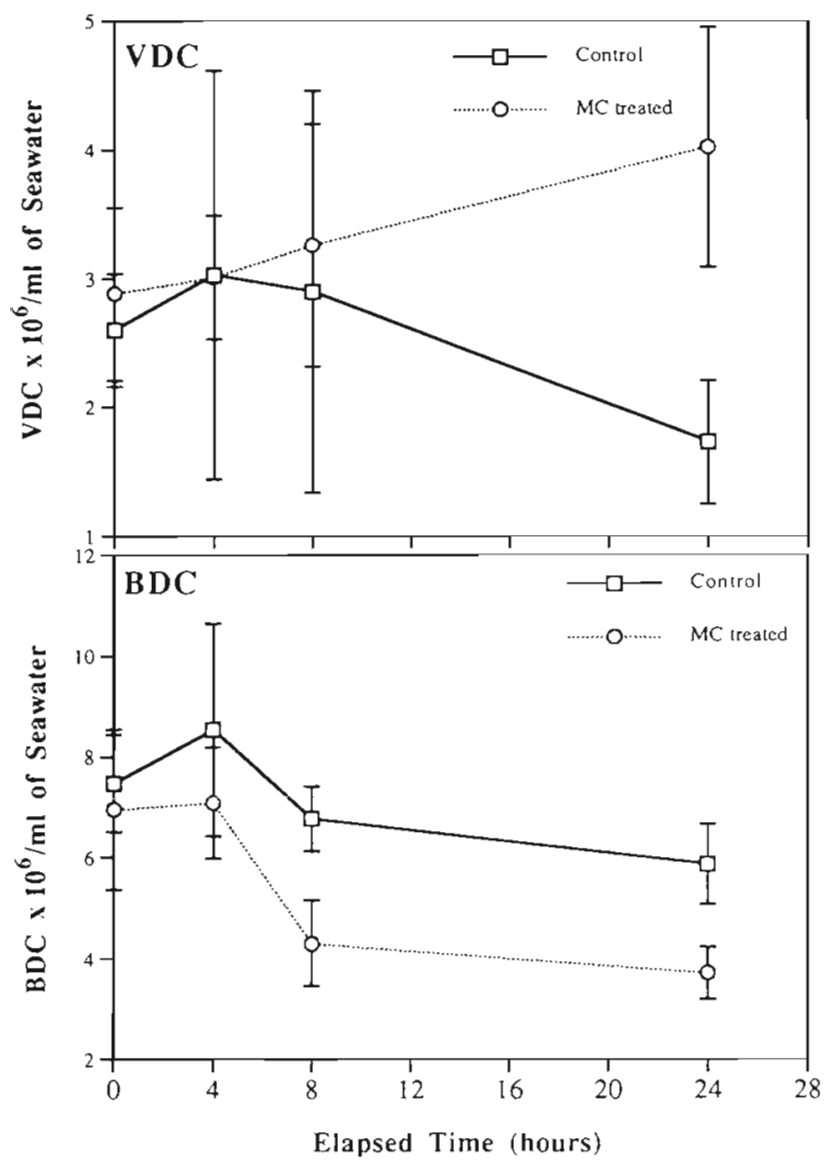

Fig. 4. Time series sampling of a mitomycun C-treated water sample and a control from Atlantic Stn 1. Error bars generated as in Fig. 2

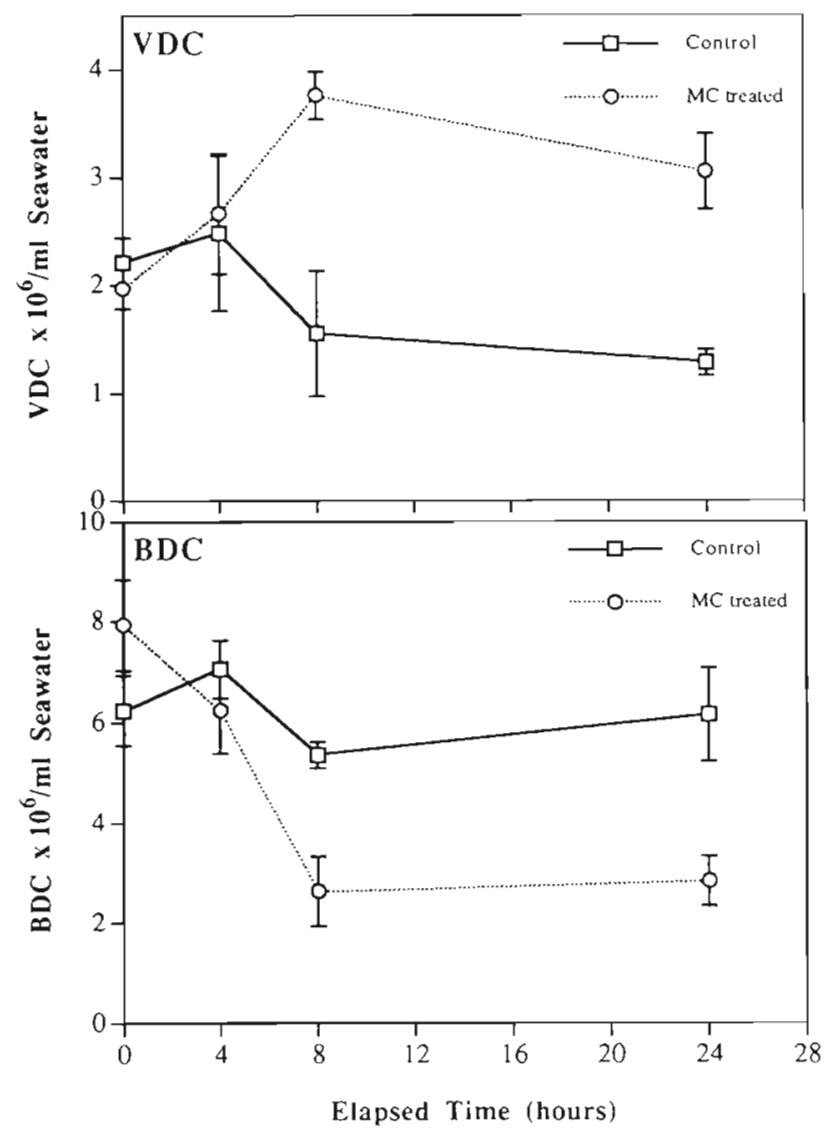

Fig. 5. Time series sampling of a mitomycin C-treated water sample and a control from Atlantic Stn 5. Error bars generated as in Flg. 2

\section{Induction time series}

The results of 2 time series studies for samples collected from Atlantic Stn 1 and Stn 5 are shown in Fig. 4 \& Fig. 5, respectively. For the sample from Atlantic Stn 1 , an increase in VDC (from $2.9 \times 10^{6}$ to $4.0 \times 10^{6}$ $\mathrm{ml}^{-1}$ ) was detected $24 \mathrm{~h}$ after the addition of mitomycin C. VDC decreased in the control sample (from $2.6 \times 10^{6}$ to $1.7 \times 10^{6} \mathrm{ml}^{-1}$ ). BDC started to decrease after $4 \mathrm{~h}$ of incubation in both the mitomycin C-treated and control samples. The mitomycin $\mathrm{C}$-treated sample had a significantly lower BDC than the control sample after $8 \mathrm{~h}$ of incubation. No dramatic changes in BDC were found after $8 \mathrm{~h}$.

At Atlantic Stn 5, VDC increased significantly 8 h after the addition of mitomycin $\mathrm{C}$, from $2.6 \times 10^{6} \mathrm{ml}^{-1}$ to $3.8 \times 10^{6} \mathrm{ml}^{-1}$, in the mitomycin C-treated sample. VDC decreased to $1.5 \times 10^{6} \mathrm{ml}^{-1}$ in the control sample. A slight decrease of VDC from 8 to $24 \mathrm{~h}$ was detected in both treated and control samples, but neither were statistically significant. BDC decreased in both treated and control samples after $8 \mathrm{~h}$ into the experiment. 
Table 5. Abundance of lysogenic bacteria in a variety of marine environments, averages in parentheses

\begin{tabular}{|c|c|c|c|c|}
\hline Environment & $\begin{array}{l}\text { No. of } \\
\text { samples } \\
\text { tested }\end{array}$ & $\begin{array}{l}\text { No. of } \\
\text { samples } \\
\text { induced }\end{array}$ & $\begin{array}{c}\% \text { lysogenic } \\
\text { Bacteria } \\
\text { mortality }\end{array}$ & $\begin{array}{c}\text { cteria based on } \\
\text { Average } \\
\text { burst size }(30)\end{array}$ \\
\hline $\begin{array}{l}\text { Eutrophic } \\
\text { estuarine }\end{array}$ & 10 & 8 & $\begin{array}{c}10.5-67.3 \\
(34)\end{array}$ & $\begin{array}{l}2-38 \\
(13)\end{array}$ \\
\hline Coastal & 5 & 3 & $\begin{array}{c}26.5-78.3 \\
(54)\end{array}$ & $\begin{array}{c}1.5-3.5 \\
(2.8)\end{array}$ \\
\hline $\begin{array}{l}\text { Oligotrophic } \\
\text { offshore }\end{array}$ & 11 & 3 & $\begin{array}{c}13.2-25 \\
(18)\end{array}$ & $\begin{array}{r}2.5-7 \\
(4.5)\end{array}$ \\
\hline Total & 26 & 14 & $\begin{array}{c}10.5-78.3 \\
(35)\end{array}$ & $\begin{array}{c}1.5-38 \\
(8.8)\end{array}$ \\
\hline
\end{tabular}

environments. However, these estimates were significantly higher than those generated from the average burst size (1.5 to $38 \%$, average: $8.8 \%$ ), suggesting an important portion of bacterial mortality may due to toxic effects of the inducing agent. The greatest toxic effects were always found in polyaromatic hydrocarbon (PAH) induced samples.

Table 6 summarizes the efficiency of chemical and physical treatments used for the induction of indigenous lysogens. PAH chemicals, including phenanthrene, naphthalene and pyrene, were the most efficient agents $(73 \%)$ for the induction of marine lysogens. UV radiation and mito-

Table 6. Efficiency of inducing agents for the induction of indigenous marine lysogens

\begin{tabular}{|lccc|}
\hline $\begin{array}{l}\text { Inducing } \\
\text { agent }\end{array}$ & $\begin{array}{c}\text { No. of expts } \\
\text { performed }\end{array}$ & $\begin{array}{c}\text { No. of samples } \\
\text { induced }\end{array}$ & $\begin{array}{c}\text { Efficiency } \\
(\%)\end{array}$ \\
\hline Mitomycin C & 18 & 7 & 39 \\
UV radiation & 12 & 5 & 42 \\
Sunlight & 9 & 0 & 0 \\
Temperature & 6 & 2 & 33 \\
Pressure & 3 & 0 & 0 \\
PAH chemicals & 11 & 8 & 73 \\
Bunker C fuel oil & 6 & 0 & 0 \\
Trichloroethylene & 6 & 1 & 17 \\
\hline
\end{tabular}

However, the decrease in the mitomycin C-treated sample was much more dramatic, from $6.2 \times 10^{6} \mathrm{ml}^{-1}$ to $2.8 \times 10^{6} \mathrm{ml}^{-1} \mathrm{BDC}$ in the treated sample were significantly lower than those in the control sample after $8 \mathrm{~h}$ of incubation.

Table 5 summarizes data for the abundance of lysogens in a variety of marine environments. The number of samples which indicated the presence of lysogens is based on the positive results of one or more induction methods in a sample. In eutrophic estuarine and coastal environments, 80 and $60 \%$, respectively, of the samples tested were positive for lysogens as indicated by prophage induction. Only $27 \%$ of the samples from oligotrophic offshore environments were induced by our criterion. However, 2 of the 11 samples showed significant increases in phage numbers without decreases in bacterial density and therefore were not considered positive lysogens by our criteria. The percentage of lysogenic bacteria in natural bacterial communities as estimated by the difference in bacteria number between control and induced sample ranged from 10.5 to $67.3 \%$ (average: $34 \%$ ) in estuarine environments, 26.5 to $78.3 \%$ (average: $54 \%$ ) in coastal environments, and 13.2 to $25 \%$ (average: $18 \%$ ) in oligotrophic offshore mycin $\mathrm{C}$ also had relatively high induction efficiencies (42 and 39\%, respectively). Sunlight had no effect on the induction of marine lysogenic bacteria for all the samples tested in this study. Increased temperature caused induction in 2 of the 6 experiments, yet this efficiency is based on only a few samples tested by this method.

\section{DISCUSSION}

\section{Induction of indigenous lysogenic bacteria from the marine environment}

A total of 26 stations from a diversity of marine environments were examined for the presence of inducible lysogens in this study. Samples from 14 stations $(53.8 \%)$ showed prophage induction by one or more of the methods employed (Table 5). We are not surprised to see that many of the samples failed to respond to an inducing agent, because some of the inducing agents tested are only known to induce prophage from cultured lysogenic bacteria. These agents and/or the concentrations used may either kill the natural bacterial cormmunity before induction occurs or not be sufficient enough to cause the induction. Additionally, different strains of bacteria in the natural community may respond to these treatments very differently.

It should also be noted that the detection of inducible lysogens in this study was based on a simultaneous statistically significant increase in VDC and decrease in BDC. Other studies have only used an increase in VDC as an indication of prophage induction (C. A. Suttle pers. comm.). This criterion would add 2 more environments (Gulf of Mexico Stn 9 and Atlantic Stn 6x) to our list of environments yielding a positive response. Therefore, criteria used in this study may underestimate the indigenous lysogenic population, because induction may occur even when there are only small 
changes in total VDC and $\mathrm{BDC}$ or because the changes in VDC and BDC cannot be detected by the stringent statistical tests. A problem in these studies is the variability in VDC, which prevents statistical significance even when a numerically greater VDC is encountered as a result of induction. For example, 22 of 26 samples contained numerically greater VDC after induction. If our precision had been greater in VDC, these might all be significant. This is particularly a problem in offshore environments, where low viral abundance generates a greater standard division in TEM VDC. Thus we may have considerably underestimated the number of environments yielding a positive response.

In a previous study we have shown that about $43 \%$ of marine bacterial isolates contained inducible prophage or bacteriocins when treated with mitomycin $C$ (Jiang \& Paul 1994). In the present study, the percentage of lysogens among the total indigenous bacteria varies over a wide range. The percentage of bacteria that were lysogenic for all positively induced samples ranged from 1.5 to $38 \%$ (average: $8.8 \%$ ), as determined by the average burst size. We have also attempted to estimate the proportion of lysogens by determining the bacterial mortality caused by inducing agents, assuming all mortality was due to induced viral lysis. This method yielded a higher percentage of lysogens than the average viral burst size method, particularly for coastal and offshore environments. The best agreement occurred for the estuarine environmental samples.

There are problems with both these methods of estimating the percentage of the lysogenic populations. The direct measurement of decrease in bacterial counts assumes that this decrease is only the result of lysis by induced prophage after correcting from control samples for lytic-phage-caused mortality, mortality by grazing and senescent cell death. This method ignored mortality caused by toxicity of the inducing agent. Yet, all the inducing agents used in this study are mutagenic or DNA-damaging agents, and may cause the mortality of indigenous bacteria without induction. The mortality caused by toxicity was particularly great for all xenobiotic hydrocarbon-treated samples, as well as for coastal and offshore samples induced by mitomycin $\mathrm{C}$. The later microbial populations are probably particularly sensitive to foreign chemicals, and perhaps lower concentrations of inducing agents should have been used there. Moreover, bacteria might also be lysed by bacteriocins in the treated incubations without virus production. The toxicity of these inducing agents to the non-lysogenic bacteria and the occurrence of bacteriocin induction may explain why the percenatge of mortality in the induced samples were significantly higher than the number of induced lysogens as determined by the average burst size.
Problems also existed when an average burst size was used for the estimation of the percentage of lysogenic populations. In our opinion, there is not a good method for the determination of bacteriophage burst size in natural populations of marine bacteria. The results obtained by counting electron-dense particles inside bacteria under high-voltage TEM tend to bias toward bacteria containing 6 or more electron-dense particles and ignore those containing fewer particles. This method will also count aggregated viruses as bursting bacterial cells. Phage particles attached to the top surface of bacteria may also be counted as mature particles inside a cell. High burst sizes may lead to the underestimation of the presence of lysogens in bacterial communities.

In the study of marine bacterial isolates, we found that the percentage of lysogenic bacterial isolates increased from nearshore environments to of fshore environments (Jiang \& Paul 1994). However, this was not found for indigenous marine bacteria, as very few inductions were observed in samples from oligotrophic offshore environments. There may be several explanations for this phenomenon: (1) indigenous bacteria in eutrophic estuarine environments may be more metabolically active than their counterparts in oligotrophic environments. Induction of prophage in cultured bacteria is more efficient during the active exponential growth, because of the active replication and DNA repair mechanisms occurring during such growth (Ackermann \& DuBow 1987). Therefore, bacteria in eutrophic environments would be more likely to be induced. (2) Bacteria in oligotrophic environments may be more sensitive to the toxicity of the inducing agents. Toxic molecules in estuarine environmental samples may be bound by dissolved organic matter (DOM), detritus and other suspended particles in the sample. Additionally, estuarine bacteria are exposed to more pollutants and may be less sensitive to the toxic effects of the agents. Therefore, at the same final concentration of inducing agent, oligotrophic bacteria may be killed before induction occurs. Decreases in VDC and $B D C$ were often found at the same time in treated oligotrophic samples. (3) VDC in the oligotrophic environments are about 1 order of magnitude lower than those in the estuarine environment, resulting in greater variability as described above.

The efficiency of mitomycin $C$ and UV radiation $(254 \mathrm{~nm})$ as inducing agents in cultured lysogenic bacteria has been well documented (Ackermann \& DuBow 1987). In this study, we showed that these were also efficient agents for the direct induction of indigenous marine lysogenic bacteria. However, these treatments will not occur naturally in the marine environment. Very little UV radiation at $<300 \mathrm{~nm}$ wavelength occurs in the natural sunlight (Schrader et al. 1994). Sunlight could not 
induce indigenous lysogenic bacteria (Table 6). Prophage induction occurs when the host cell is subjected to mutagenic agents, DNA damaging agents, or stress conditions which trigger the 'SOS' response in the bacterial cell thereby removing the prophage transcription repressor (Ackermann \& DuBow 1987). To understand the environmental factors that may cause the prophage induction in the marine environment, we tested several agents and conditions which can be found in the marine environment, including stress conditions such as elevated temperature and pressure, and mutagenic agents such as aromatic and aliphatic hydrocarbons as inducing agents. Although all these agents have been documented as inducing agents for cultured lysogenic bacteria (Ackermann \& DuBow 1987), no work has been done on their ability to induce marine lysogens. In this study, 2 of the 6 experiments performed with elevated temperature in different samples resulted in induction. Some $\lambda$ phage are known to have temperature sensitive promoters. The prophage repressor protein is sensitive to temperatures of $>40^{\circ} \mathrm{C}$, at which its structure conformationally changes and no longer binds to the promoter (Bertani 1953, Birge 1994). These results suggests that short-term temperature increases may be 1 of the factors for lysogenic induction in the marine environment. Of the 2 temperatures employed, 30 and $42^{\circ} \mathrm{C}$, the former occurs in surface waters in tropical and subtropical environments, whereas the latter might be encountered in shallow tide pools in the tropics or near hydrothermal vents. Experiments with increased pressure were performed 3 times with coastal and oligotrophic water samples, and none showed lysogenic induction by our criteria. However, no induction was detected in 2 samples even when mitomycin $C$ was used, suggesting that these samples had low levels of lysogens. More research is needed to understand the effect of pressure on the induction of indigenous lysogens in the marine environment.

PAHs were found to be the most efficient inducing agents for the indigenous lysogenic bacteria (Table 6). This not surprising because PAHs are known carcinogens and mutagens, which cause induction of the 'SOS' DNA repair response (Moreau et al. 1976). Thus, environments which contain mutagenic pollutants may have a large degree of prophage induction occurring. This result confirmed our original hypotheses that xenobiotic pollutants may be important agents in the induction of lysogenic bacteria in the marine environment. One may argue that Bunker $\mathrm{C} \# 6$ fuel oil, which contains all the PAHs yet did not cause the induction of any of the environmental samples, contradicts that logic. We think that the concentration of Bunker $\mathrm{C} \# 6$ fuel oil added to the samples may have been too high, causing the mortality of the microbial population before inducing the lysogenic induction.
All the previous studies we have performed included a 16 to $24 \mathrm{~h}$ incubation. Our time series analysis suggested that induction could be detected even after $4 \mathrm{~h}$, with statistical significance after $8 \mathrm{~h}$ incubation. Further incubation may cause a decrease of both viruses and bacteria in the sample.

Lysogeny may impart a competitive advantage to marine bacteria over non-lysogens in the marine environment. Lysogens of Escherichia coli have been shown to reproduce more rapidly than non-lysogens during aerobic growth in nutrient-limited chemostats (Edlin et al. 1975, 1977, Lin et al. 1977). The majority of the environments in the ocean are nutrient-limited environments; thus lysogens may be more fit than the corresponding non-lysogens in these environments. Lysogens can also benefit from phage conversion, which is the expression of phenotype characters encoded by prophage. Prophage are known to encode the gene for antibiotic resistance (Smith 1972), cytotoxin (Hayashi et al. 1990) and other virulence factors (Barondess \& Beckwith 1990). These characters may increase the survival rate and competitive fitness of lysogens in the environment. The most basic phageconversion character is homoimmunity, which protects the bacterium from lytic infection. In the marine environment where viral abundance exceeds bacterial abundance by a factor of 10 , homoimmunity may contribute significantly to the survival of lysogenic bacteria.

In summary, lysogenic bacteria may be an important portion of the marine bacterial community. Many important environmental factors including xenobiotic pollutants and sudden temperature changes may be inducing agents for natural lysogenic viral production in the marine environment. Whether such phage production contributes significantly to bacterial mortality of sensitive hosts or such cell lysis contributes to the production of DOM remains unknown.

Acknowledgements. We are grateful to Chris Kellogg for her valuable suggestions and comments in the revision of this manuscript, and to Pam Sutton and Ted Van Vleet for providing us with aromatic and aliphatic hydrocarbons. This research was supported by NSF grants OCE 9115942 and OCE 9502775, and also by a Gulf Oceanography Fellowship and a Knight Fellowship to S.C.J

\section{LITERATURE CITED}

Ackermann HW, DuBow MS (1987) Viruses of prokaryotes, Vol 1 General properties of bacteriophages. CRC Press, Boca Raton

Barondess JJ, Beckwith J (1990) A bacterial virulence determinant encoded by lysogenic coliphage $\lambda$. Nature 346 : $871-873$

Bergh $\varnothing$, Børsheim KY, Bratbak G, Heldal M (1989) High abundance of viruses found in aquatic environments. Nature 340:467-468 
Bertani G (1953) Lysogenic versus lytic cycle of phage multiplication. Cold Spring Harbour Symp Quant Biol 18:65-70

Birge EA (1994) Bacterial and bacteriophage genetics, 3rd edn. Springer-Verlag, New York

Borsheim KY (1993) Native marine bacteriophages. FEMS Microbiol Ecol 102:141-159

Bratbak G. Heldal , M, Norland S, Thingstad TF (1990) Viruses as partners in spring bloom microbial trophodynamics. Appl Environ Microbiol 56:1400-1405

Bratbak G. Heldal M, Thingstad TF, Riemann $B$, Haslund $\mathrm{OH}$ (1992) Incorporation of viruses into the budget of microbial C-transfer. A first approach. Mar Ecol Prog Ser 83: $273-280$

Cottrell M. Suttle CA (1995) Dynamics of a lytic virus infecting the photosynthetic marine picoflagellate Micromonas pusilla. Limnol Oceanogr 40:730-739

Edlin G, Lin L, Bitner R (1977) Reproductive fitness of P1, P2, and Mu lysogens of Escherichia coli. J Virol 21:560-564

Edlin G, Lin L, Kudrna R (1975) Lysogens of E. coli reproduce more rapidly than non-lysogens. Nature 255:735-737

Eganhouse RP, Calder JA (1976) The solubility of medium molecular weight aromatic hydrocarbons and the effects of hydrocarbon co-solutes and salinity. Geochim Cosmochim Acta 40:555-561

Freifelder D (1987) Molecular biology. Jones \& Bartlett Inc, Boston

Fuhrman JA, Noble RR (1995) Viruses and protists cause similar bacterial mortality in coastal seawater Limnol Oceanogr 40:1236-1242

Fuhrman JA, Suttle CA (1993) Viruses in marine planktonic systems. Oceanography 6:57-63

Hayashi T, Baba T, Matsumoto H. Terawaki Y (1990) Phageconversion of cytotoxin production in Pseudomonas aeruginosa. Mol Microbiol 4:1703-1709

Heldal M. Bratbak G (1991) Production and decay of viruses in aquatic environments. Mar Ecol Prog Ser 72:205-212

Jiang SC, Paul JH (1994) Seasonal and diel abundance of viruses and occurrence of lysogeny/bacterlocinogeny in the marine environment. Mar Ecol Prog Ser 104:163-172

Jiang SC. Thurmond JM, Pichard SL, Paul JH (1992) Concentration of microbial populations from aquatic environments by Vortex Flow Filtration. Mar Ecol Prog Ser 80: $101-107$

This article was presented by S. Y. Newell (Senior Editorial Advisor), Sapelo Island, Georgia, USA
Levin BR, Lenski RE (1983) Coevolution in bacteria and their viruses and plasmids. In: Futuyma DJ. Slatkin M (eds) Coevolution. Sinauer, Sunderland, p 99-127

Lin L, Bitner R, Edlin G (1977) Increased reproductive fitness of Escherichia coli Lambda lysogens. J Virol 21:554-559

May WE, Wasik SP (1978) Determination of the solubility behavior of some polycyclic aromatic hydrocarbons in water. Anal Chem 50:997-1000

Moreau P, Bailone A, Devoret R (1976) Prophage $\lambda$ induction in Escherichia coli K12 envA uvrB: a highly sensitive test for potential carcinogens. Proc Natl Acad Sci 73: $3700-3704$

Paul JH (1982) The use of Hoechst dyes 33258 and 33342 for the enumeration of attached and pelagic bacteria. Appl Environ Microbiol 43:939-949

Paul JH, Jiang SC, Rose JB (1991) Concentration of viruses and dissolved DNA from aquatic environments by vortex flow filtration. Appl Environ Microbiol 57:2107-2204

Schrader JO, Lufburrow MD, Kokjohn TA (1994) Effects of stress on the replication potential of bacteriophages. 94th Gen Meet Am Soc Microbiol, Las Vegas, Abstract N213

Smith HW (1972) Ampicillin resistance in Escherichia coli by phage infection. Nat New Biol 238:205-206

Tapper MA. Hicks RE (1994) Ultraviolet light and chemical induction of temperate bacteriophage from a Great Lake ecosystem. 94th Gen Meet Am Soc Microbiol, Las Vegas, Abstract N211

Thingstad TF, Heldal M, Bratbak G, Dunda I (1993) Are viruses important partners in pelagic food webs? Trends Ecol \& Evol 8:209-213

Weinbauer MG, Fuks D, Puskaric S, Peduzzi P (1995) Diel, seasonal and depth-related variability of viruses and dissolved DNA in the northern Adriatic sea. Microb Ecol 30: $25-41$

Weinbauer MG, Peduzzi P (1995) Significance of viruses versus heterotrophic nanoflagellates for controlling bacterial abundance in the northern Adriatic sea. J Plankton Res 17 : $1851-1856$

Wilcox RM, Fuhrman JA (1994) Bacterial viruses in coastal seawater: lytic rather than lysogenic production. Mar Ecol Prog Ser 114:35-45

Zar JH (1984) Biostatistical analysis, 2nd edn. Prentice-Hall Inc, Englewood Cliffs

Manuscript first received: March 1, 1996

Revised version accepted: July 22, 1996 